\title{
A 2018 Update on the Editorial and Publication Policy of Seminars in Thrombosis and Hemostasis
}

\author{
Emmanuel J. Favaloro, PhD, FFSc (RCPA) ${ }^{1}$ \\ ${ }^{1}$ Department of Haematology, Sydney Centres for Thrombosis and \\ Haemostasis, Institute of Clinical Pathology and Medical Research, \\ Westmead Hospital, Westmead, NSW, Australia
}

Semin Thromb Hemost 2018;44:307-311.

This Editorial is meant to update the readership, potential contributors to Seminars in Thrombosis and Hemostasis (STH), potential reviewers of articles submitted to STH, and the STH Editorial Board on the journal's editorial and publication policy.

STH publishes in both print and online. The online version of STH, as well as general information about the journal, author information/instructions, journal scope, Editorial Board membership, and information on the Eberhard $\mathrm{F}$ Mammen awards (Young Investigator and Most Popular), resides at (or can easily be navigated from) https://www.thieme-connect.com/products/ejournals/journal/10.1055/s-00000077. As stated on the website, the journal's 'Aims and Scope' is reflected by the following statement:

"Seminars in Thrombosis and Hemostasis is a topic driven review journal that focuses on all issues related to hemostatic and thrombotic disorders. As one of the premiere review journals in the field, Seminars in Thrombosis and Hemostasis serves as a comprehensive forum for important advances in clinical and laboratory diagnosis and therapeutic interventions. The journal also publishes a limited number of peer-reviewed original research papers.

Seminars offers an informed perspective on today's pivotal issues, including hemophilia A \& B, thrombophilia, gene therapy, venous and arterial thrombosis, von Willebrand disease, platelet disorders, vascular disorders, and thromboembolic diseases. Attention is also given to the latest developments in pharmaceutical drugs along with treatment and current management techniques. The journal also periodically publishes sponsored supplements to further highlight emerging trends in the field."

As also noted on the website, STH is indexed in Journal Citation Reports (JCR), Emerging Sources Citation Index (ESCI), Biol Abstracts, BIOSIS, Chem Abstracts, Current Contents/Clinical Medicine, Current Contents/LS, EMBASE, Excerpta Medica, Index Intern de Cardiologia, Index Medicus,

Address for correspondence E.J. Favaloro, Department of Haematology, Institute of Clinical Pathology and Medical Research (ICPMR), Westmead Hospital, Westmead, NSW, 2145, Australia (e-mail: emmanuel.

favaloro@health.nsw.gov.au).
MEDLINE, Reference Update, Research Alert, Science Citation Index, SCOPUS, and Scisearch.

STH has been publishing papers since 1974, initially under the leadership of its founding Editor-in-Chief (EIC), Eberhard F Mammen, who sadly passed away in 2008 , at which time I took over the reins as EIC. Some of this history was published as part of our 40th-year anniversary celebrations in $2014 .^{1}$ STH started with four issues per year, publishing around 300 to 400 print pages per year. STH grew to six issues per year in 1996, then publishing around 500 to 700 pages per year. Another change in 2006 saw STH publishing eight issues per year and around 800 to 1000 pages per year. Every year since then, STH has continued with eight issues and around 800 to 1000 print pages.

As a topic-driven review journal, STH primarily publishes themed issues (meaning an issue of STH is given a specific theme, and then theme-related content is sourced (or solicited) from internationally recognized experts in the field. However, such a model best fits a print-based journal, and since most content for STH (as well as most journals these days) is accessed electronically, the theme-based content, albeit still our main focus, is no longer all that STH publishes. Accordingly, STH also publishes 'non-themed' issues, inclusive of unsolicited material and has been doing so for some time. Indeed, the current issue line up approximates five to seven 'themed' issues per year and two to three 'nonthemed' issues per year. The so-called 'non-themed' issues are better known as 'compilation' issues. The purpose of these 'compilation' issues is to publish material of interest to our readership that does not otherwise fit a 'themed issue' in progress. This can still comprise solicited material (typically reviews), but also includes submissions from our Young Investigator Award winners, as well as unsolicited material submitted to STH for publication and accepted after peerreview, and when these do not otherwise fit a 'themed issue' in progress. The compilation issues are currently compiled by
Copyright @ 2018 by Thieme Medical Publishers, Inc., 333 Seventh Avenue, New York, NY 10001, USA. Tel: +1(212) 584-4662.
DOI https://doi.org/ 10.1055/s-0038-1654718. ISSN 0094-6176.
Thrombosis and Hemostasis -

Part III; Guest Editor: Sam

Schulman, MD, PhD. 
Senior Editors of STH, being myself with professor Giuseppe Lippi (the so called 'Editorial Compilation' series, which currently sits at 'Part V') ${ }^{2}$ and another compilation series currently compiled by professor Sam Schulman (the so called 'Recent Advances in Thrombosis and Hemostasis' series, which currently sits at 'Part III'). ${ }^{3}$ Although the material published in the compilation series does not relate to a specific 'theme', the content still, of course, relates to the general theme of thrombosis and hemostasis.

Included in each issue of STH is a variety of material, but primarily reviews. As noted in the 'Aims and Scope', STH will also publish occasional original material, of course, still subject to peer review, but this is in the minority as compared with reviews. Indeed, in preparation for this Editorial, I assessed the content that STH published in the years 2015 to 2017 inclusive and could identify that the percentages for each year approximated 80 to $90 \%$ for 'reviews' as compared to 10 to $20 \%$ for 'original studies' (-Fig. 1). STH aims to publish in the order of 80 to 100 full-length papers per year, which therefore averages out to some 10 to 12 full-length papers per issue. In addition, each issue has a Preface, which explains the purpose of the issue (the context around the 'theme'), as well as a summary of the issue content and other relevant background. STH also publishes occasional correspondence (as 'Letters to the Editor'); these usually are in response to, or comment on, previous papers published in STH, but may alternatively reflect discussion around another topic of interest, a case report(s), or other material of potential interest to the readership. Sometimes, a manuscript is submitted as a 'review' or an 'original study', but does not in the view of the EIC/Editorial Board/reviewers warrant publication in STH as a full-length paper, and the authors may be offered the opportunity to resubmit the paper or elements thereof as correspondence, or else the authors are advised to seek publication in another journal. Other material included in STH includes Editorials (such as this one), Meeting Reports, Erratum, and Publisher Information.

In July 2017, the Editorial Board of STH met formally at the last congress (Berlin ISTH2017) of the International Society on Thrombosis and Hemostasis (ISTH). This meeting represented the largest gathering of the Editorial Board in my memory and was very constructive. One of the proposals put forward at the meeting was that STH reconsider its policy in regards to publication of original material. Indeed, this matter caused vigorous debate, with some Board members having strong views against such publications, and others having equally strong views in support of such publications. One of the concerns raised was the opinion that there was perception among the readership and potential authors that such material was not peer reviewed. On the contrary, the readership and all potential authors should be assured that all material published in STH is peer reviewed. It is true that most material is peer reviewed by members of the STH Editorial Board, the number of which current sits at 38 , but this is not expectedly different to other international journals. However, whenever needed (e.g., if members of the STH Editorial Board identify conflicts or deficiencies in

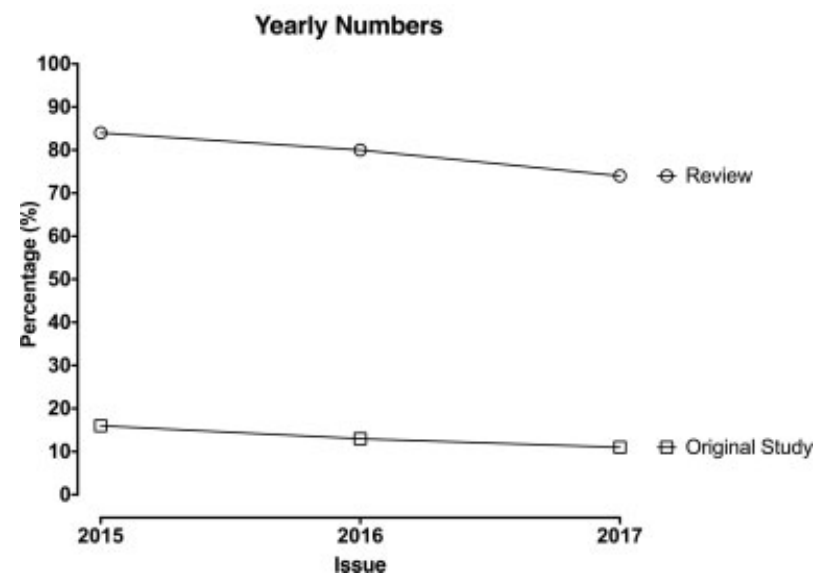

Fig. 1 Proportion of 'reviews' as compared to 'original studies' published in Seminars in Thrombosis and Hemostasis during the past 3 years (2015-2017). In brief, the percentage of 'original studies' for each year has been consistently between 10 and $20 \%$ of the total fulllength papers published.

regards to their ability to undertake effective reviews), external reviewers are additionally or alternatively sourced.

Some members of the Editorial Board also reflected additional viewpoints, including one in which the word 'Seminars', as encapsulated in our journal name, portrayed the concept of 'reviews' and not 'original studies'. While it can be agreed that the term 'seminars' largely reflects the idea of discursive text, as captured within reviews, the term should not be considered as being limited to 'reviews'. Essentially, any written text that contains discussion and narration of ideas, and that 'teaches the audience', can fulfil the definition of 'seminar'. Historically, STH has always published original articles. Indeed, in some of the very early issues published by this journal, some contained more original studies than reviews. Thus, the founding EIC, Eberhard F Mammen, also considered that STH should publish more than just reviews. Importantly, several original studies are in the top 10 listing of the most highly cited papers of STH, including one by Eberhard F Mammen. ${ }^{4}$

It was also recognized among the STH Editorial Board that some of the content published within STH did not easily sit as either an 'original study' or a 'review', with 'composite' or 'hybrid' papers, and perhaps other categories of papers, also published on occasion. For example, 'hybrid' papers may take the form of (i) a review or guidance document that includes some 'original material' as examples for guidance and (ii) a series of case studies that also includes a formal literature review component.

Irrespective, the decision was taken at the 2017 Editorial Board meeting to formally survey Editorial Board members for their support or otherwise of continued publication of original studies, and if so, whether limits and/or extra oversight be applied. In brief, the voting was close, but the majority Editorial Board decision was to (i) continue to publish original studies, but (ii) to restrict the number of published original studies to a small proportion of the total 
(here, it was agreed to cap these as $<15$ to $20 \%$ of the total of full-length papers) and (iii) to ensure additional oversight of such submissions, including at least one extra review external to the STH Editorial Board. It is interesting to me that the majority Editorial Board decision was to cap original studies to $<15$ to $20 \%$ of the total of full papers, given that this essentially matches recent trends (-Fig. 1). What this means in practice, however, given a total limit of 80 to 100 full papers per year, is that STH will need to limit the number of original full-length studies published in STH to around less than 15 such papers per year. This will expectantly result in a high 'rejection rate' for original studies, which generally represent unsolicited material. I would make formal apologies to prospective authors for this in advance, but all respected journals have a limit to the quantity of papers they can publish.

In addition, as part of the preparation for this Editorial, I reviewed the recent data for unsolicited manuscripts submitted for publication in the past 2 years and can inform the readership that STH has declined to publish over $80 \%$ of unsolicited original studies. In comparison, STH declined to publish around $50 \%$ of the unsolicited review articles submitted for publication.

However, this 'formalization' of the STH policy around the publication of original studies is not forever set in stone. There was also Editorial Board agreement to review the policy in around 2 years and to try to gauge the success of this policy in the interim. Key to this is an understanding around whether the published original studies are actually of interest to the STH readership. Thus, if the STH readership supports continued publication of original studies, original studies should be published in STH. So, how can we gauge the value of original studies ( $v s$ reviews) in STH? Well, we have at least two markers of quality that we use for STH. The first relates to download data, as a measure of 'readership popularity', and the second relates to citation index, as a measure of 'scientific relevance.' STH publishes annual Editorials around these metrics, with the last such Editorials published earlier in 2018. ${ }^{5,6}$ It needs to be recognized that such metrics are available for analysis some 2 years after papers are published; therefore, the success or otherwise of papers published in 2018 will not be known until 2020 or so.

Although not wishing to pre-empt the findings of our future analysis in a few years, I did none-the-less briefly review the latest available download data. ${ }^{5}$ Here it also needs to be recognized that this metric is an imperfect marker, since material that is 'free to download,' as opposed to material available only to STH subscribers, will always predominate on the top download lists. Thus, although only some 10 to $20 \%$ of the STH content is free to download, this free to download material comprises some $70 \%$ of the top 100 download list. Thus, nearly $100 \%$ of the Editorials and Prefaces that STH published in 2015 and 2016 appeared in the most recent top 100 download list (reflecting the most downloaded material of 2016 and 2017 inclusive). ${ }^{5}$ More relevant is that $\sim 10 \%$ of the original studies published between 2015 and 2017 inclusive appeared on the list, compared with $\sim 10 \%$ of the reviews published in the same period. Thus, certainly there is no evidence that original studies are currently of less interest to our readership than reviews are. Similarly, an interim review of citation data identified four original studies as compared to 26 reviews in the top 30 cited papers published in STH in 2015 and 2016. Given that relative percentage publications in the same period are 29 and 164, respectively, this equates to $4 / 29$ (13.8\%) of original studies as compared to $26 / 164$ (14.6\%) of reviews. Again, there is no evidence that original studies represent substantially less citation potential than reviews. However, these metrics will be formally re-assessed in around 2 years.

Given that STH has decided, via an Editorial Board vote, to continue to publish original studies, albeit at $<20 \%$ of the total of full papers, then additional thought needs to go into this process in the future. Clearly, there is always some discordance between the relative content of individual issues of STH. It is expected that 'Themed' issues will continue to be mostly comprised of review articles. This makes perfect sense since these comprise solicited material, and it would be most unusual to solicit an original study. Generally, the occasional original study appearing in a themed issue of STH is more likely to have been an unsolicited manuscript that matches the theme of the issue and is accepted for publication after peer review. In contrast, as the major component of unsolicited material submitted for publication in STH comprises original studies rather than reviews, it will expectedly mean that the compilation issues will often have a proportion of original material content that is higher than $20 \%$. Indeed, the current compilation issue of STH in which this Editorial appears is a good example of this expectation.

As also previously noted, it is sometimes difficult to be black and white about whether a given paper is a review, an original study, a hybrid of both, or indeed something else. As a follow-up to the initial vote of the Editorial Board, I constructed another survey in which I selected eight papers recently published in STH. I asked the Editorial Board to characterize these eight papers as a review, an original study, a hybrid of both, or other material (i.e., something else). It was interesting that from these four choices, none of the eight papers achieved a $100 \%$ consensus of viewpoints. Certainly, those papers which were 'clearly' in my mind an original study were also identified as being an original study by the majority of the Editorial Board; similarly, those papers which were 'clearly' in my mind a review were also seen as being a review by the majority of the Editorial Board. However, what was largely 'black and white' to me, and presumably 'black and white' to each other member of the board, in composite, finished up being various shades of gray. In particular, one paper, a systematic review and metaanalysis was variably identified as a review (45\% of respondents), an original study (21\%), a hybrid of both (24\%), and other (10\%; generally including a comment that 'meta-analysis' should represent a third separate category).

Therefore, although the current compilation issue of STH comprises 10 papers, and a higher proportion of 'original material' than $20 \%$, I would agree with the assessment of the 


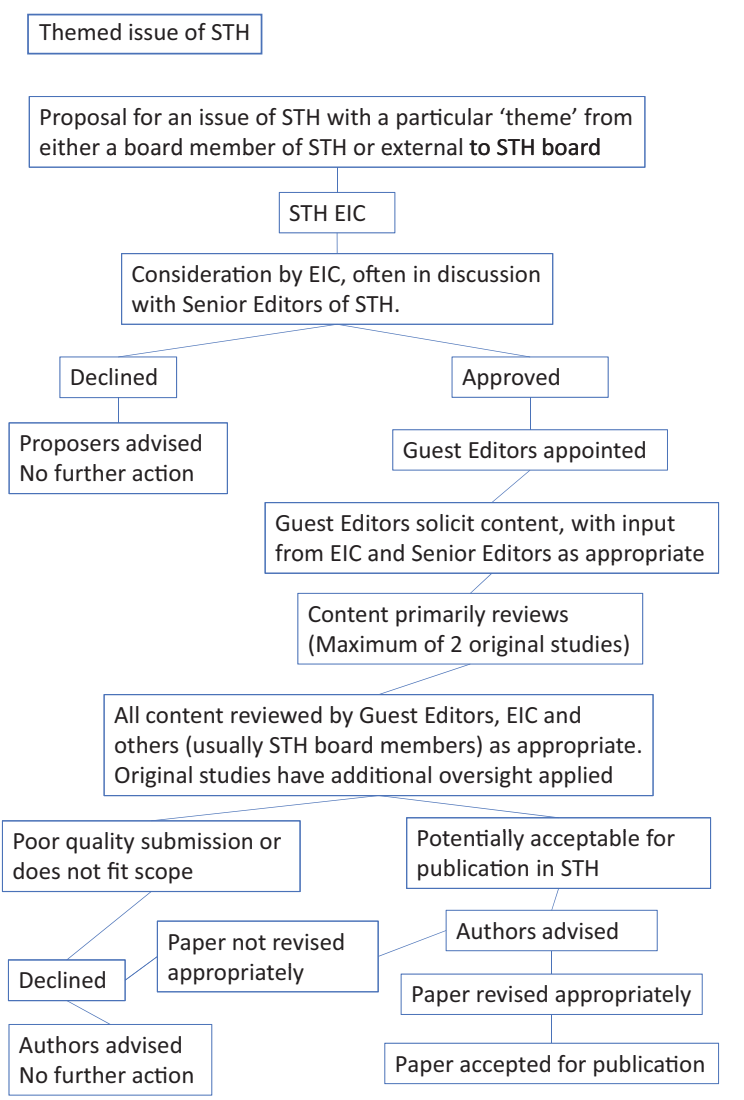

Fig. 2 A flow chart that summarizes how 'Themed' issues of STH are developed and compiled. The processes typically begins with a proposal sent to the EIC, inclusive of proposed content, although the EIC may alternatively also propose an issue for consideration of the STH Editorial Board members or other external experts. The EIC usually discusses themes to be developed with the Senior Editors of STH, and also periodically with the remainder of the Editorial Board. The initial proposal may or may not proceed. If progressed, Guest Editors are invited or appointed, and if inexperienced are given instructions on how to proceed. These Guest Editors handle the process of content (invitation of potential contributors, initial receipt and review of contributions, requests for revisions, etc.). The EIC and/ or Senior Editors will provide guidance for the process and additional oversight and manuscript review as appropriate. Additional reviews may be sought from other members of the STH Editorial Board or externally. All 'original studies' submitted and further considered for publication now require additional oversight, including at least one additional review from a non-STH Board member. There will also be a maximum of two original papers permitted in each 'Themed' issue. EIC, Editor-in-Chief; STH, Seminars in Thrombosis and Hemostasis.

Guest Editor ${ }^{3}$ that the 10 papers comprise: three that are purely original research (30\%), two that are a combination of original research and literature reviews ('hybrids'; $20 \%$ ), three that are review articles (30\%), and two that are systematic reviews and meta-analyses (20\%).

Finally, I would like to clarify, for the sake of the readership, the Editorial Board, prospective authors, and reviewers, the STH publication process as I see it, and this is depicted as flow diagrams in -Figs. 2 and $\mathbf{3}$. These flow diagrams reflect the way that manuscripts are handled by STH, and how 'themed' and 'non-themed' ('compilation') issues are

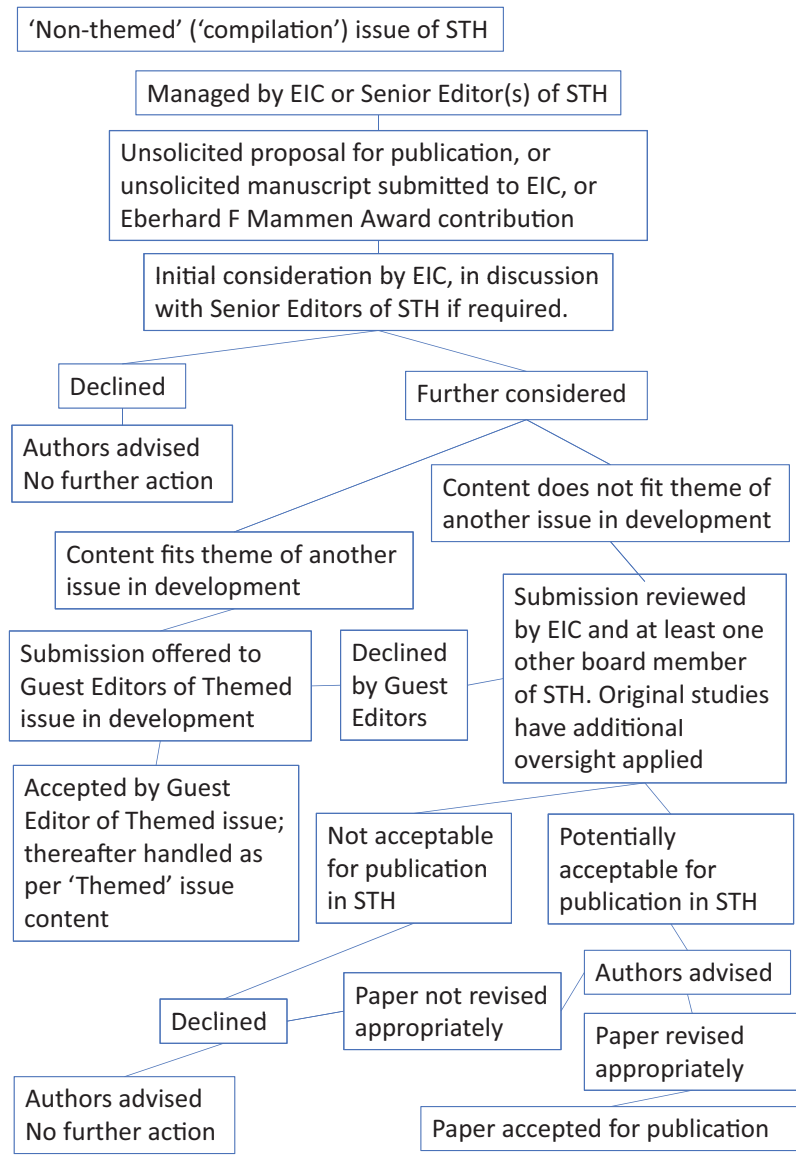

Fig. 3 A flow chart that summarizes how 'non-themed' ('composite') issues of ST) are developed and compiled. These typically comprise either the best of unsolicited manuscripts submitted for possible publication in STH, material submitted from winners of the Eberhard $F$ Mammen Young Investigator Award as a condition of their award, or other content that may be solicited by the EIC or a senior Editorial Board member of STH (where considered submissions do not fit a themed issue in progress). For the composite issues, the EIC and/or a senior Editorial Board member of STH will act as the senior Guest Editors, with others also potentially invited or appointed as required to assist with manuscript handling and review. The Guest Editors again handle the process (including initial receipt and review of contributions, requests for revisions, etc.). Additional reviews may be sought from other members of the STH Editorial Board or externally, and all 'original studies' submitted and further considered for publication now require additional oversight, including at least one additional review from a non-STH Board member. There is no formal limit to the number of original papers permitted in each non-themed issue, other than by consideration of the limit to the total number of original studies permitted to be published in each year (i.e., original articles need to comprise $<20 \%$ of total of full papers published in each year), which the EIC will monitor. However, it is expected that as a general guide, even the 'non-themed' compilation issues will usually comprise a greater number of review articles than original papers. EIC, Editor-inChief; STH, Seminars in Thrombosis and Hemostasis

assembled. Moreover, - Table 1 summarizes the current 'targets' for STH.

Any unsolicited proposal or unsolicited manuscript submitted to STH will be initially screened by the EIC, often in consultation with the Senior Editors. These may be declined at this stage and without further review for a variety of 
Table 1 Current targets for Seminars in Thrombosis and Hemostasis

- Eight issues per year (typically 5-6 as 'themed' issues and 2-3 as 'non-themed' compilation issues)
- Minimum of 80 and maximum of 100 full papers published each year (thus, each issue to contain on average $~ 10$ to 12 full papers).
- Original studies to comprise a minimum of the total of full length papers (target $<15 \%$; maximum $<20 \%$ )
- Reviews (including systematic reviews) to comprise a maximum of the total of full length papers (target $>80 \%$ )
- Meta-analyses to be considered in similar manner to reviews
- Hybrid papers (i.e., those containing a mixture of review/guidance and original material) will be treated as per the majority content
(e.g., those predominantly considered to be review/guidance with minor element of original material will be treated as a review)
- Other material (e.g., Meeting Reports and Correspondence/Letters to the Editor) may be accepted at the discretion of the EIC.
- eProofs for accepted papers to be sent to authors within a maximum of 3 months of finalization/acceptance of manuscript by STH
- Accepted papers to appear on eFirst (online publication version) within a maximum of 4 months of finalization/acceptance of
manuscript by STH and/or within a target of 1 month of finalization of eProof.

Abbreviations: EIC, Editor-in-Chief; STH, Seminars in Thrombosis and Hemostasis.

reasons, including (but not limited to) (i) does not match the journal scope; (ii) not considered of sufficient quality for publication in STH; (iii) duplicates material recently published in STH; (iv) following consideration of publication quota limits (applies in particular to original studies); (v) high level of text duplication identified after duplication check; and (vi) any other reason as seems appropriate to the journal. Given the limits applied to the number of papers, especially ‘original studies' that STH can publish, this process will by necessity disappoint a large proportion of prospective contributors to STH. For this, our sincere apologies in advance. On the other hand, this process will expectantly continue to generate published material of high quality, including occasional high-quality original studies that will ultimately reward our readership.

\section{References}

1 Favaloro EJ. A short history of Thrombosis and Hemostasis: part I (40th year celebratory issue). Semin Thromb Hemost 2014; 40(05):521-525

2 Favaloro EJ, Lippi G. Editorial compilation V. Semin Thromb Hemost 2018;44(03):193-196

3 Schulman S. Recent advances in thrombosis and hemostasis-part III. Semin Thromb Hemost 2018;44(04):312-314

4 Mammen EF, Comp PC, Gosselin R, et al. PFA-100 system: a new method for assessment of platelet dysfunction. Semin Thromb Hemost 1998;24(02):195-202

5 Favaloro EJ. Welcome to Seminars in Thrombosis \& Hemostasis 2018. New (2016) Impact Factor and Most Highly Cited Papers. Semin Thromb Hemost 2018;44(01):1-4

6 Favaloro EJ. Editorial - 2018 Eberhard F Mammen award announcements: part I - most popular articles. Semin Thromb Hemost 2018;44(03):185-192 